\title{
Power Coordinates and the Bicircular Quartic.
}

\author{
By R. Wilson, M.A.
}

\section{(Received 2nd December 1924. Read 17th January 1925.)}

\section{Introduction.}

* Casey, in an extensive and complete mémoire, has discussed very thoroughly the bicircular quartic. Among the unfinished notes of $+D r$ Clifford the properties of the curve are established by means of a tetracyclic system of coordinates. Such a system of coordinates has been extensively used by $\$$ Coolidge. The object of this paper is to present a valid discussion of the curve in terms of a tricyclic system of "power" coordinates.

\section{The Circle Orthogonal to Three Given Circles.}

Given three fixed non-coaxal circles, whose powers with regard to a point are $S_{1}, S_{2}, S_{3}$, where $S_{k}=x^{2}+y^{2}+2 g_{k} x+2 f_{k} y+c_{k}$, the point is uniquely determinate when the ratios $\left(S_{1}: S_{3}: S_{3}\right)$ are given. These are the power coordinates of the point.

The equation of any circle of the doubly-infinite system orthogonal to the radical circle $C$ of $S_{1}, S_{2}, S_{3}$ is $l S_{1}+m S_{2}+n S_{3}=0$. The tangential coordinates of this circle are $(l: m: n)$. We shall find the equation of the circle $C$.

The point-circles of the system, since they are orthogonal to $C$, lie on $C$. Hence $C$ is the locus or envelope of the point circles of the system, and all point-circles of the system must satisfy the equation

$$
\text { or } \begin{gathered}
\left(l g_{1}+m g_{2}+n g_{3}\right)^{2}+\left(l f_{1}+m f_{2}+n f_{3}\right)^{2}=\left(l c_{1}+m c_{2}+n c_{3}\right)(l+m+n) \\
\Sigma l^{2}\left(g_{1}^{2}+f_{1}^{2}-c_{1}\right)+\Sigma m n\left(2 g_{2} g_{3}+2 f_{2} f_{3}-c_{2}-c_{3}\right)=0 .
\end{gathered}
$$

Analytically, $r_{1}$, the radius of $S_{1}$, is defined by $r_{1}^{2}=g_{1}^{2}+f_{1}^{2}-c_{1}$, and $\psi_{23}$, the angle at which $S_{2}, S_{3}$ cut, by

$$
2 r_{2} r_{3} \cos \psi_{23}=2 g_{2} g_{3}+2 f_{2} f_{3}-c_{2}-c_{3} \text {. }
$$

* CAsey - Trans. Royal Irish Academy. Dublin, 1867.

+ W. K. Clurford-Mathematical Papers.

$¥$ Coolidat-The Circle and the Sphere. Oxford, 1918. 
Hence the tangential equation of $C$ is

$$
\Sigma l^{2} r_{1}^{2}-2 \Sigma m n r_{2} r_{3} \cos \psi_{23}=0
$$

Reciprocally, the equation of $C$ in power coordinates is

$$
\Sigma \frac{S_{1}^{2}}{r_{1}{ }^{2}} \sin ^{2} \psi_{23}+2 \Sigma \frac{S_{2} S_{3}}{r_{2} r_{3}}\left(\cos \psi_{31} \cos \psi_{12}+\cos \psi_{23}\right)=0 \ldots \ldots
$$

Since $S_{1}, S_{2}, S_{3}$ in this equation represent the powers of any point on $C$ with regard to the three circles, we have obtained a generalisation of Ptolemy's Theorem as will be verified later.

The discussion is analytical, and holds whether the circles considered are real or not. Three types of circles will arise, as follows :-

Coefficients of $S$ real, centre real, radius real, circle referred to as real.

Coefficients of $S$ real, centre real, radius imaginary, circle referred to as imaginary.

Coefficients of $S$ complex, circle referred to as complex.

If $S_{1}, S_{3}, S_{3}$ form an orthogonal system, equations (1) and (2) simplify. $\quad S_{1}, S_{2}, S_{3}, S_{4}\left(C \equiv S_{4}\right)$ then form a system of four mutually orthogonal circles, and each one is the locus of the pointcircles formed from the other three.

The equation of $S_{4}$ in power coordinates is then

$$
S_{1}{ }^{2} / r_{1}{ }^{2}+S_{2}{ }^{2} / r_{2}{ }^{2}+S_{3}{ }^{2} / r_{3}{ }^{2}=0 \text {. }
$$

Every point on $S_{4}$ satisfies $S_{4}=0$ and $S_{1}{ }^{2} / r_{1}{ }^{2}+S_{2}{ }^{2} / r_{2}{ }^{2}+S_{3}{ }^{2} / r_{3}{ }^{2}=0$, and by symmetry similar results hold when $S_{n}, S_{2}, S_{3}, S_{4}$ are permuted cyclically.

Hence we have the identity

$$
S_{1}^{2} / r_{1}^{2}+S_{4}^{2} / r_{2}^{2}+S_{3}^{2} / r_{3}^{2}+S_{4}^{2} / r_{4}^{2}=0
$$

The tangential equation of $S_{4}$ is $r_{1}^{2} l^{2}+r_{3}^{2} m^{2}+r_{3}^{2} n^{2}=0$.

Cor.-If the circles are point-circles,

$$
g_{k}{ }^{3}+f_{k}{ }^{2}-c_{k}=0 \quad(K=1,2,3) .
$$

The tangential equation of $C$ becomes $d_{23}{ }^{2} m n+d_{32}{ }^{2} n l+d_{12}{ }^{2} l m=0$, where $\left.d_{h k}^{2}=\left(g_{h}-g_{k}\right)^{2}+f_{h}-f_{k}\right)^{2}$. Call these three base point-circles $C_{1}, C_{2}, C_{3}$. Then the circle $C$, passing through $C_{1}, C_{2}, C_{3}$ is $\Sigma d_{23}{ }^{4} C_{1}{ }^{2}-2 \Sigma d_{31}{ }^{2} d_{12}{ }^{2} C_{2} C_{3}=0$, or

$$
d_{2} \quad \sqrt{C_{1}} \pm d_{31} \sqrt{C_{2}} \pm d_{12} \sqrt{C_{3}}=0
$$

which is Ptolemy's Theorem. 


\section{The Nature of the Orthogonal System of Base Circles.}

For real points, from (3), one at least of the four circles is nonreal. Two of these circles cannot be imaginary. For if $S_{1}, S_{2}$ are so, since they are also orthogonal $\left(g_{1}-g_{2}\right)^{2}+\left(f_{1}-f_{2}\right)^{2}=r_{1}{ }^{2}+r_{2}^{2}$, where $g_{s}, f_{s}$ are real, and $r_{13}{ }^{2} r_{2}^{2}$ both negative. This is clearly impossible.

Defining $\Sigma_{k}{ }^{2} \equiv S_{k}{ }^{2} / r_{k}{ }^{2}$, equation (3) becomes

$$
\Sigma_{1}^{2}+\Sigma_{2}^{2}+\Sigma_{i j}^{2}+\Sigma_{4}^{2}=0
$$

For real points it follows from (5), that if one circle be complex and the other two real, the fourth must be conjugate complex.

It is easy to prove that the four circles cannot be two pairs of conjugate complexes. We shall show later that for a bicircular quartic with real coefficients, the complex orthogonal circles occur in pairs.

\section{The Bicircular Quartic.}

The equation $(a, b, c, f, g, h)\left(S_{1}, S_{2}, S_{3}\right)^{2}=0$ represents a bicircular quartic. A comparison of coefficients shows that any bicircular quartic can be thrown into this form.

The foci are point-circles having double contact with the curve. Hence if $l S_{1}+m S_{2}+n S_{3}=0$ is a focus,

$$
\left.\begin{array}{l}
A l^{2}+B m^{2}+C n^{2}+2 F m n+2 G n l+2 H l m=0 \text { and } \\
r_{1}^{2} l^{2}+r_{2}^{2} m^{2}+r_{3}^{2} n^{2}-2 r_{2} r_{3} \cos \psi_{23} m n^{-2} r_{3} r_{1} \cos \psi_{31} n l-2 r_{1} r_{2} \cos \psi_{12} l m=0
\end{array}\right\}
$$

The two equations determine four sets of mutual ratios $l: m: n$, and therefore four foci lying on the circle orthogonal to $S_{1}, S_{21} S_{3}$.

The equations in power-coordinates corresponding to (6) are

$$
\left.\begin{array}{l}
a S_{1}^{2}+b S_{2}^{2}+c S_{3}^{2}+2 f S_{2} S_{3}+2 g S_{3} S_{1}+2 h S_{1} S_{2}=0 \text { and } \\
\frac{\sin ^{2} \psi_{2}}{r_{2}^{2}} S_{1}^{2}+\ldots+2 \frac{\left(\cos \psi_{31} \cos \psi_{12}+\cos \psi_{23}\right)}{r_{2} r_{3}} S_{2} S_{3}+\ldots=0
\end{array}\right\}
$$

There is a unique linear transformation

$$
S_{k}=p_{k} S_{1}^{\prime}+q_{k} S_{2}^{\prime}+r_{k} S_{3}^{\prime},(k=1,2,3)
$$

for which equations (7) take the canonical form

$$
\left.\begin{array}{r}
a^{\prime} S_{1}^{\prime 2}+b^{\prime} S_{2}^{\prime 2}+c^{\prime} S_{3}^{\prime 2}=0 \\
S_{1}^{\prime 2} / r_{3}^{\prime 2}+S_{2}^{\prime 2} / r_{2}^{\prime 2}+S_{3}^{\prime 2} / r_{3}^{\prime 2}=0
\end{array}\right\}
$$

$S_{1}^{\prime}, S_{2}^{\prime}, S_{:}^{\prime}$ belong to the same system orthogonal to the radical 
circle of $S_{1}, S_{3}, S_{3}$. Further, in the transformed equations,

$$
\cos \psi_{31}^{\prime} \cos \psi_{12}^{\prime}+\cos \psi_{23}^{\prime}=0 \text {, etc. }
$$

and it is easy to show that the only solution of these equations for which cotresponding forms (8) exist is given by $\cos \psi^{\prime}{ }_{k k}=0$, $\sin \psi^{\prime}{ }_{h k}=1$. Hence $S_{1}^{\prime}, S_{2}^{\prime}, S_{3}^{\prime}$ are orthogonal, and the four foci given by the transformed equations (6) lie on the radical circle $S_{\downarrow}^{\prime}$.

Since $S_{1}^{\prime}, S_{2}^{\prime}, S_{3}^{\prime}, S_{4}^{\prime}$ form a mutually orthogonal system,

$$
S_{1}^{\prime 2} / r_{1}^{\prime 2}+S_{2}^{\prime 2} / r_{2}^{\prime 2}+S_{3}^{\prime 2} / r_{3}^{\prime \prime 2}+S_{4}^{\prime \prime 2} / r_{4}^{\prime 2} \equiv 0 \text {, }
$$

and the substitution in (8) from this equation yields symmetrical results for each of $S_{1}^{\prime}, S_{2}^{\prime}, S_{3}^{\prime}, S_{4}^{\prime}$. Hence the sixteen foci lie in fours on the four circles.

\section{The Uniqueness of Solution.}

Suppose the quartic to be reduced to the canonical form in two systems $a S_{1}{ }^{2}+b S_{2}{ }^{2}+c S_{3}{ }^{2}=0, a^{\prime} S_{1}{ }^{2}+b^{\prime} S_{2}{ }^{\prime 2}+c^{\prime} S_{3}{ }^{\prime 2}=0$,

where $\quad S_{1}, S_{2}, S_{3}, S_{4} ; S_{1}^{\prime}, S_{2}^{\prime \prime}, S_{3}^{\prime}, S_{4}^{\prime}$ form two mutually orthogonal sets of circles, none of which coincide. If this is so, the foci can be distributed in four concyclic sets of four in two ways.

Any circle is $l S_{1}+m S_{3}+n S_{3}+p S_{4}=0$. Hence we can transform the second system in terms of $S_{1}, S_{2}, S_{3}, S_{4}$, viz., $l_{k} S_{1}+m_{k} S_{2}+n_{k} S_{3}+p_{k} S_{4}=\left(l_{k}+m_{k}+n_{k}+p_{k}\right) S_{k},(k=1,2,3)$, giving \ $\left\{a^{\prime}\left[\left(l_{1} S_{1}+m_{1} S_{2}+n_{1} S_{3}+p_{1} S_{4}\right) /\left(l_{1}+m_{1}+n_{1}+p_{1}\right)\right]^{2}\right\}=\Sigma_{a} S_{1}{ }^{2}$, etc $\ldots$

From these we obtain the sets of equations

$$
\begin{aligned}
& \Sigma\left[a^{\prime} l_{1}^{2} /\left(l_{1}+m_{1}+n_{1}+p_{1}\right)^{2}\right]=a,(l, m, n) \text { and } \\
& \searrow\left[a^{\prime} p_{1}^{3} /\left(l_{1}+m_{1}+n_{1}+p_{1}\right)^{2}\right]=0 ; \\
& Y\left[a^{\prime} m_{1} n_{1} /\left(l_{1}+m_{1}+n_{1}+p_{1}\right)^{2}\right]=0,(l, m, n) \text { and } \\
& บ\left[a^{\prime} l_{1} p_{1} /\left(l_{1}+m_{1}+n_{1}+p_{1}\right)^{2}\right]=0,(l, m, n) .
\end{aligned}
$$

There are four linear homegeneous equations in

$$
\begin{aligned}
& a^{\prime} p_{1} /\left(l_{1}+m_{1}+n_{1}+p_{1}\right)^{2} \text { etc.... } \\
& \text { Hence either } p_{1}=p_{2}=p_{3}=0 \text { or }\left\|\begin{array}{lll}
l_{1} & l_{2} & l_{3} \\
m_{1} & m_{2} & m_{3} \\
n_{1} & n_{2} & n_{3} \\
p_{1} & p_{2} & p_{3}
\end{array}\right\|=0 \text {. }
\end{aligned}
$$

This last condition is easily seen to be impossible. Therefore $p_{1}=p_{2}=p_{3}=0$, and $S_{1}^{\prime}, S_{2}^{\prime}, S_{3}^{\prime}$, are circles of the system $l S_{1}+m S_{2}+n S_{3}=0$, orthogonal to $S_{4}$. Hence $S_{4} \equiv S_{4}$. 
This produces the identities, true for all points on the curve

$$
a S_{1}{ }^{2}+b S_{2}{ }^{2}+c S_{3}{ }^{2} \equiv a^{\prime} S_{1}^{\prime 2}+b^{\prime} S_{2}^{\prime 2}+c S_{3}^{\prime 2}
$$

and $S_{1}{ }^{2} / r_{1}{ }^{2}+S_{2}{ }^{2} / r_{2}{ }^{2}+S_{3}{ }^{2} / r_{3}{ }^{2} \equiv-S_{4}{ }^{2} / r_{4}{ }^{2} \equiv S_{1}^{\prime}{ }^{2} / r_{1}{ }^{2}+S_{2}^{\prime 2} / r_{2}{ }^{2}+S_{3}^{\prime}{ }^{\prime 2} / r_{3}{ }^{2}$. It is easily shown that these can only hold if

$$
S_{1} \equiv S_{1}^{\prime}, S_{2} \equiv S_{2}^{\prime}, S_{3} \equiv S_{3}^{\prime} \text {. }
$$

Hence the solution is unique.

VI. The Foci.

Putting $S_{k}{ }_{k}{ }^{2} / r^{\prime}{ }_{k}{ }^{2} \equiv \sum_{k}{ }^{2}$ as in (5) the curve is $\Sigma_{1}{ }^{2} / \alpha+\Sigma_{2}{ }^{2} / \beta+\sum_{j}{ }^{2} / \gamma-0$, where $\Sigma_{1}{ }^{2}+\Sigma_{2}{ }^{2}+\Sigma_{3}{ }^{2}+\Sigma_{4}{ }^{2} \equiv 0$ and equations (8) become

$$
\left.\begin{array}{l}
\Sigma_{1}{ }^{2} / \alpha+\Sigma_{2}{ }^{2} / \beta+\Sigma_{3}{ }^{2} / \gamma=0 \\
\Sigma_{1}{ }^{2}+\Sigma_{2}{ }^{2}+\Sigma_{3}{ }^{2}=0
\end{array}\right\}
$$

By means of the identity, we can express the curve in terms of any three of the four coordinates, giving four similar forms of the equation.

The tangential forms corresponding to (9) are

$$
\left.\begin{array}{rl}
\alpha \lambda^{2}+\beta \mu^{2}+\gamma v^{2} & =0 \\
\lambda^{2}+\mu^{2}+\nu^{2} & =0
\end{array}\right\} \text { giving } \frac{\lambda^{2}}{\beta-\gamma}=\frac{\mu^{2}}{\gamma-\alpha}=\frac{\nu^{2}}{\alpha-\beta},
$$

which determine four non-singular foci $\lambda \Sigma_{1}+\mu \Sigma_{3}+\nu \Sigma_{3}=0$ lying on $\Sigma_{4}$.

We tabulate the results as follows :-

Foci $\sqrt{\alpha-\beta} \Sigma_{2} \pm \sqrt{\gamma-\alpha} \Sigma_{3} \pm \sqrt{\beta-\gamma} \Sigma_{t}=0$ lying on $\Sigma_{1}$

$$
\begin{aligned}
& \sqrt{\beta-\gamma} \Sigma_{3} \pm \sqrt{\gamma-\alpha} \Sigma_{4} \pm \sqrt{\alpha-\beta} \Sigma_{1}=0 \\
& \sqrt{\alpha-\beta} \Sigma_{1} \pm \sqrt{\gamma-\alpha} \Sigma_{1} \pm \sqrt{\beta-\gamma} \Sigma_{2}=0 \\
& \sqrt{\beta-\gamma} \Sigma_{1} \pm \sqrt{\gamma-\alpha} \Sigma_{2} \pm \sqrt{\alpha-\beta} \Sigma_{3}=0
\end{aligned}
$$

$$
\left.\begin{array}{l}
\Sigma_{1}, \\
\Sigma_{n}, \\
\Sigma_{3}, \\
\Sigma_{n}
\end{array}\right\}
$$

This determines the sixteen non-singular foci.

The coefficients in every case are $\pm \sqrt{\beta-\gamma} \pm \sqrt{\gamma-\alpha,} \pm \sqrt{\alpha-\beta}$. Hence all the confocal curves are given by the one parameter system $\Sigma_{1}{ }^{2} \alpha_{\alpha+\theta}+\Sigma_{2}^{2} / \beta+\theta+\Sigma_{3}^{2} / \gamma+\theta=0$.

We a re concerned with the bicircular quartic of real coefticients. Suppose the four base circles to be complex. Then the conjugates $\Sigma_{1}^{\prime}, \Sigma_{2}^{\prime}, \Sigma_{3}^{\prime}, \Sigma_{4}^{\prime}$ must also form an orthogonal system, for the equations of orthogonality still hold when the imaginary part cbanges sign. Since the curve has only real coefficients it can be written in the conjugate form $\Sigma_{1}^{\prime 2} / a_{a^{\prime}}+\Sigma_{2}^{\prime 2} /_{\beta^{\prime}}+\Sigma_{3}^{\prime 2} /_{\gamma^{\prime}}=0$. From 
the uniqueness of solution this must be one of the four forms (9). Hence $\Sigma_{1}^{\prime}, \Sigma_{2}^{\prime}, \Sigma_{3}^{\prime}, \Sigma_{4}^{\prime}$ must be $\Sigma_{1}, \Sigma_{2}, \Sigma_{3}, \Sigma_{4}$ in some order, and the complex base circles occur in pairs.

Applying the results of $\$$ III., it is clear that for a bicircular quartic of real coefficients two types only are possible, as follows:-

The base circles are (a) three real, one imaginary; $(b)$ two real, two conjugate complex.

In case (a), equations (10) show that there are four real foci only, all lying on one of the real circles.

In case $(b)$ let $\Sigma_{2}, \Sigma_{3}$ be conjugate complexes, and $\Sigma_{1}, \Sigma_{\downarrow}$ real.

Then in the equation of the quartic $\beta, \gamma$ are conjugate complexes and $\alpha$ is real.

Write $\alpha=a, \beta=b+\iota c, \gamma=b-\imath c, \Sigma_{2}=R e^{\imath \phi}, \Sigma_{8}=R e^{-\imath \phi}, R, \phi$, being functions of $x, y$.

The foci are easily found to be $\Sigma_{\jmath} \pm r^{\frac{1}{3}} e^{l \phi} \frac{\phi \phi}{2} R e^{\iota \varphi} \pm r^{\frac{1}{3}} e^{-\frac{-\tau}{2}} R e^{-\imath \phi}$, where $r e^{\iota \phi}=-\frac{1}{2}+\frac{\iota(a-b)}{2 c}$.

The foci are real only for both signs positive or both negative. Hence two real and two unreal foci lie on $\Sigma_{\downarrow}$. (10) shows that for the foci on $\Sigma_{1}$, the coefficients of $\Sigma_{2}, \Sigma_{z}$ are interchanged. This will produce real foci for the case only of different signs. Hence two real and two unreal foci lie on $\check{\Sigma}_{1}$. (10) shows that the foci lying on $\Sigma_{2}, \Sigma_{3}$ are unreal.

\section{Fundamental Point Circles.}

If we take three foci as base circles, three sets of mutual ratios defining the foci are $(1: 0: 0),(0: 1: 0),(0: 0: 1)$. The tangential equation (6) gives

$$
F \mu \nu+G v \lambda+H \lambda \mu=0
$$

and hence $A=B=C=0$.

The equation of the quartic becomes

$$
\begin{aligned}
& \left(-F^{2},-G^{2},-H^{2}, G H, H F, F G\right)\left(C_{1}, C_{2}, C_{3}\right)^{2}=0 \\
& \text { or } \pm \sqrt{F C_{1}} \pm \sqrt{G C_{2}} \pm \sqrt{H C_{3}}=0 \text {, i.e. } \sqrt{F} \cdot r_{1} \pm \sqrt{G} \cdot r_{2} \pm \sqrt{H} \cdot r_{3}=0 \text {, }
\end{aligned}
$$
where $r_{1}, r_{2}, r_{3}$ are the distances of any point on the quartic from any three foci. From (4) the equation of the orthogonal circle passing through $C_{1}, C_{2}, C_{3}$ is

$$
\left(d_{23}^{2}, d_{31}^{2}, d_{12}^{2},-d_{31} d_{132}-d_{18} d_{28},-d_{23} d_{21}\right)\left(C_{1}, C_{2}, C_{3}\right)^{2}=0 \text {. }
$$


The corresponding tangential equation is

$$
d_{23} \mu \nu+d_{31} \nu \lambda+d_{12} \lambda \mu .=0 .
$$

This equation together with (11) has four solutions,

$$
(1: 0: 0),(0: 1: 0),(0: 0: 1)
$$

and

$$
\lambda\left(G d_{12}-H d_{31}\right)=\mu\left(H d_{23}-F d_{12}\right)=\nu\left(F d_{31}-G d_{23}\right) .
$$

Hence the fourth concyclic focus is the point circle

$$
\Sigma C_{1} /\left(G d_{12}-H d_{31}\right)=0 \text {. }
$$

\section{The Circular Cubic.}

The equation $(a, b, c, f, g, h)\left(S_{1}, S_{2}, S_{3}\right)^{*}=0$ represents a circular cubic if $a+b+c+f+g+h=0$. The point $S_{1}=S_{2}=S_{3}$, hence lies on the curve. So, in the canonical form $\alpha \Sigma_{1}{ }^{2}+\beta \Sigma_{2}{ }^{2}+\gamma \Sigma_{3}{ }^{2}=0$, $\alpha / r_{1}{ }^{2}+\beta / r_{2}{ }^{2}+\gamma / r_{3}{ }^{2}=0$, and the centres of the four base circles lie on the cubic. With this additional condition, the results of $\S \S$ III.-VI. hold for the circular cubie.

In the form $(a, b, c,-\sqrt{b c},-\sqrt{c a},-\sqrt{a b})\left(C_{1}, C_{2}, C_{3}\right)^{2}=0$ we have $a+b+c-2 \sqrt{b c}-2 \sqrt{c a}-2 \sqrt{a b}=0$ or $\pm \sqrt{a} \pm \sqrt{b} \pm \sqrt{c}=0$.

The equation to such cubic has the forms

$$
\left(\sqrt{C_{2}}-\sqrt{C_{3}}\right) / \sqrt{a}= \pm\left(\sqrt{C_{3}}-\sqrt{C_{1}}\right) / \sqrt{b}= \pm\left(\sqrt{C_{1}}-\sqrt{C_{2}}\right) / \sqrt{c}
$$

If $r_{1}, r_{2}, r_{3}$ be the distances of any point of the cubic from any three foci, we have $\frac{r_{2}-r_{3}}{\sqrt{a}}= \pm \frac{r_{3}-r_{1}}{\sqrt{b}}= \pm \frac{r_{1}-r_{2}}{\sqrt{c}}$

\section{A System of Curves.}

Suppose we have two curves $(a, b, c, f, g, h)\left(S_{1}, S_{2}, S_{3}\right)^{2}=0$,

$$
\left(a^{\prime}, b^{\prime}, c^{\prime}, f^{\prime}, g^{\prime}, h^{\prime}\right)\left(S_{1}^{\prime}, S_{2}^{\prime}, S_{3}^{\prime}\right)^{2}=0 \text {. }
$$

In general it will not be possible to refer these two curves to the same system of base circles, unless they each have four foci lying on the same circle, $C$.

In this case we have

$$
\begin{aligned}
& (a, b, c, f, g, h)\left(S_{1}, S_{2}, S_{3}\right)^{2} \equiv \alpha \Sigma_{1}{ }^{2}+\beta \Sigma_{2}{ }^{2}+\gamma_{3}{ }^{2} \equiv U . \\
& \left(a^{\prime}, b^{\prime}, c^{\prime}, f^{\prime}, g^{\prime}, h^{\prime}\right)\left(S_{\mathrm{r}}, S_{2}, S_{3}\right)^{2} \equiv \alpha^{\prime} \Sigma_{3}{ }^{2}+\beta^{\prime} \Sigma_{2}{ }^{2}+\gamma^{\prime} \Sigma_{3}{ }^{2} \equiv V,
\end{aligned}
$$

a unique transformation, in which $\Sigma_{1}, \Sigma_{2}, \Sigma_{3}$ are not in general 
"focal" circles. The solutions of the finite points of intersection of these two quartics are given by

$$
\left.\Sigma_{1}^{2} /\left(\beta \gamma^{\prime}-\beta^{\prime} \gamma\right)=\Sigma_{2}^{2} /\left(\gamma \alpha^{\prime}-\gamma^{\prime} \alpha\right)=\Sigma_{3}^{2} / \alpha \beta^{\prime}-\alpha^{\prime} \beta\right)
$$

or

$$
\pm \Sigma_{1} / A= \pm \Sigma_{2} / B= \pm \Sigma_{3} / C
$$

This gives the intersections of the two circles $\Sigma_{1} / A= \pm \Sigma_{2} / B$ with the two circles $\Sigma_{2} / B= \pm \Sigma_{2} / C$, and the two circles $\Sigma_{3} / C= \pm \Sigma_{1} / A$ pass through these common points. There are thus four pairs of such points, and therefore any two bicircular quartics of the form

$$
(a, b, c, f, g, h)\left(S_{1}, S_{2}, S_{3}\right)^{2}=0,\left(a^{\prime}, b^{\prime}, c^{\prime}, f^{\prime}, g^{\prime}, h^{\prime}\right)\left(S_{1}, S_{2}, S_{3}\right)^{2}=0,
$$

will have eight such connected points in common, and all such bicircular quartics possessing these properties can be thrown into the form $\alpha \Sigma_{1}^{2}+\beta \Sigma_{2}^{2}+\gamma \Sigma_{3}^{2}=0$.

The system is $U+t V$, where $t$ is a parameter.

If $\alpha+\beta+\gamma=0$, the curve is a circular cubic.

There corresponds for the case of two base cubics a system of circular cubics, and for the finite common points of these cubics condition (12) gives $\pm \Sigma_{1}= \pm \Sigma_{2}= \pm \Sigma_{3}$. These are the common points of $\Sigma_{1}= \pm \Sigma_{2}$ and $\Sigma_{2}= \pm \Sigma_{3}$, through which pass also $\Sigma_{3}= \pm \Sigma_{1}$.

This gives the point of concurrence of the three common chords and points of intersection of each common chord with a circle of the other systems, seven points in all.

The first system contains one circular cubic given by $(\alpha+\beta+\gamma)+t\left(\alpha^{\prime}+\beta^{\prime}+\gamma^{\prime}\right)=0$.

To the system $U+t V=0$ corresponds a set of invariants similar to those of the four point conic systems.

Casey has discussed these and obtained a more extended system of invariants, from the geometrical point of view. An analytical discussion of the invariant-theory from the modern point of view is to be found in Prof. Turnbull's paper.*

\section{Inversion.}

Inversion with regard to any point leaves the ratios of the coordinates $\frac{S_{k}}{r_{k}} \equiv \Sigma_{k},(k=1,2,3)$, unaltered. The inverses of the

* Proc. Royal Soc., Edinòurgh (1924), 23-50. 
base aircles are the new mutunily or orthogonal base circles $\Sigma_{k}{ }^{\prime}-\frac{S_{k}{ }^{\prime}}{r_{k}{ }^{\prime}}(k=1,2,3)$, and thus the inverse of the curve

$$
\alpha \Sigma_{1}^{2}+\beta \Sigma_{2}^{2}+\gamma \Sigma_{3}^{2} \text { is } \alpha \Sigma_{1}^{\prime 2}+\beta \Sigma_{2}^{\prime 2}+\gamma \Sigma_{3}{ }^{2}=0
$$

Inverting with regard to a point on a bicircular quartic an exception arises, for the inverse of this point is the line at infinity, the ' $S$ ' ' coordinates of any point of which are $(1: 1: 1)$.

The ' $\Sigma^{\prime}$ ' coordinates $\left(\frac{1}{r_{1}^{\prime 2}}, \frac{1}{r_{2}^{\prime 2}}, \frac{1}{r_{3}^{\prime 2}}\right)$ hence satisfy (13), i.e.

$$
\alpha / r_{1}^{\prime 2}+\beta / r_{1}^{\prime}+\gamma / r_{3}^{\prime 2}=0
$$

and the inverse curve is a circular cubic

Inversion with regard to one of the base circles leaves the system, and hence by (12), the curve, unaltered. 